\title{
Turbulent kinetic energy budgets in a model canopy: comparisons between LES and wind-tunnel experiments
}

\author{
Wusi Yue - Charles Meneveau - Marc B. Parlange • \\ Weihong Zhu • Hyung Suk Kang • Joseph Katz
}

Received: 5 September 2007 / Accepted: 15 December 2007

(C) Springer Science+Business Media B.V. 2008

\begin{abstract}
A comparative study of turbulence in a wind-tunnel model canopy is performed, using Large eddy simulation (LES) and experimental data from PIV and hot-wire anemometry measurements. The model canopy is composed of thin cylindrical stalks. In the LES, these are represented using a plant-scale approach, while the scale-dependent Lagrangian dynamic model is used as subgrid-scale model. LES predictions of turbulence statistics and energy spectra are found to be in good agreement with the experimental data. Turbulent kinetic energy (TKE) budgets from the LES simulation are analyzed to provide more information absent in the measurements. Results confirm that sloshing motions at the low levels of the canopy are mainly driven by pressure fluctuations. A difference between the energy flux obtained from the energy spectrum and the SGS dissipation rate is observed, consistent with a spectral bypass mechanism in which the real spectral flux due to cascade is smaller than that implied by the energy-spectrum level, due to direct drain by the canopy.
\end{abstract}

Keywords Wind-tunnel model canopy - Large eddy simulation · Energy spectrum . Turbulent kinetic energy budget · Particle image velocimetry $\cdot$ Hotwire anemometer

\section{Introduction}

Turbulent flow within and above plant canopies has been an important research subject since turbulence plays an important role in the transfer of momentum, heat, water vapor, carbon

W. Yue

Department of Geography and Environmental Engineering, and Center for Environmental and Applied Fluid Mechanics, The Johns Hopkins University, Baltimore, MD 21218, USA

C. Meneveau · W. Zhu · H. S. Kang · J. Katz

Department of Mechanical Engineering, and Center for Environmental and Applied Fluid Mechanics, The Johns Hopkins University, Baltimore, MD 21218, USA

M. B. Parlange $(\bowtie)$

School of Architecture, Civil and Environmental Engineering, Ecole Polytechnique Federale de Lausanne, Lausanne, CH 1015, Switzerland

e-mail: Marc.Parlange@epfl.ch 
dioxide, and other scalars between the atmosphere and plants. Turbulence inside the canopy is characterized by momentum absorption and energy dissipation by means of canopy drag throughout the whole canopy depth, leading to complex turbulence structures and high intermittency. The canopy breaks down the large-scale turbulent motions into small-scales, and the kinetic energy of the mean flow is converted into the turbulent kinetic energy (TKE) within the wakes behind plant elements $[10,44]$. Quadrant analysis shows that sweeps contribute most to the vertical momentum flux, TKE, and dissipation rate within the canopy $[45,48,49]$, contrary to that in a smooth surface layer or above a roughness layer where ejections dominate. At the lower levels of canopies, large-scale horizontal motions are observed. They do not play a dominant role in the transfer of momentum, and are called "sloshing" or inactive motions $[6,16]$. A variety of interesting large-scale turbulent coherent motions have also been observed, e.g., plant waving ("honami") in cereal crops [9], and scalar ramps (a region consisting of high concentration gradient between sweep and ejection zones) in a deciduous forest [12]. Many of the early studies are summarized and reviewed in [30] and most recently in [10]. Compared to field studies, measurements conducted in a windtunnel with a model canopy enable better control of flow conditions. Several wind-tunnel experiments with different model canopies have been carried out, e.g., model forest [23], slender cylindrical rods [33], strips [31], and model wheat [7]. Poggi et al. [28] conducted a laser Doppler anemometer experiment in a water-tunnel with fixed rods on the bottom. A more recent wind-tunnel experiment using particle image velocimetry (PIV) and hotwire anemometer (HA) techniques has been performed using a canopy consisting of cylindrical stalks $[47,49]$. In this experiment, the turbulence level has been enhanced by an active grid, achieving a Reynolds number based on the Taylor microscale of about 800 . We use the results of this experimental study in the present paper.

While many canopy turbulence characteristics have been discovered experimentally, some terms related to pressure and small-scale energy dissipation are still difficult to obtain. Pressure fluctuations are linked dynamically to the structure of the flow, specifically, turbulence at low levels in the canopy is driven by pressure fluctuations [15,35]. However, a direct experimental measurement of pressure fluctuations away from boundaries is very difficult. Three-dimensional numerical simulations have the advantage that pressure and dissipation are directly computed. Also, one can visualize detailed spatial and temporal flow structures. Early numerical investigations of turbulent canopy flows have been mostly based on the reynolds-averaged Navier-Stokes equations (RANS) [42,43], and they have had difficulties in reproducing the experimental data. The primary hurdle has been the high turbulence intermittency, which presents extra challenges in turbulence modeling.

Shaw and Schumann [34] were the first to introduce large eddy simulation (LES) to modeling of turbulent canopy flows. The plant canopy was treated as a porous body of horizontally uniform area density with constant drag coefficient, that can be classified as "field-scale" modeling. The same methodology to model the plants was subsequently used by several other investigators to study turbulence statistics and structures for a variety of canopy flows, e.g., [17,27,39,40]. Different from this traditional method, the LES performed in [46] resolved the coarse features of individual plants and field plant arrangement of a corn canopy. A local force field was used to represent an individual corn plant in this model, an approach named "plant-scale" to distinguish it from the traditional "field-scale" approach. Numerical predictions by the plant-scale approach were shown to be in better agreement with field experimental data than the field-scale approach for some important flow features. In particular, the field-scale approach overpredicted the canopy drag [46], especially at the low levels of the canopy, and excessively damped the penetration of gusts into the lower levels of the canopy. Therefore, while the field-scale approach will continue to be useful when 
one wishes to simulate extensive vegetated areas in an affordable fashion (e.g., to capture long distance transport), the plant-scale approach is preferable when simulating smaller areas to capture detailed phenomena more accurately (e.g., details of the emission or deposition processes of pollen, etc.). The objective of the present study is to validate the plant-scale LES by extensively comparing the computational predictions with the experimental wind-tunnel PIV and HA data in [49], as well as to perform a full TKE budget analysis to provide more information, not possible from the experiments. Specifically, our aim is to further clarify the relationship between pressure fluctuations and the "sloshing" motions at the lower levels of the canopy. We simulate turbulent flow within and above the wind-tunnel model canopy by means of LES based on the scale-dependent Lagrangian dynamic Smagorinsky model [4].

A brief description of the LES and the SGS model is presented in Sect. 2. The experimental setup of the model canopy is described in the same section. Section 3 presents the computational results, the comparison with the experimental data, and the TKE budget analysis. In Sect. 4, a summary of the results and conclusions are provided.

\section{Numerical simulation of wind-tunnel model canopy}

\subsection{Governing equations and SGS modeling}

The filtered incompressible Navier-Stokes equations for the present LES reads,

$$
\frac{\partial \tilde{u}_{i}}{\partial t}+\tilde{u}_{j}\left(\frac{\partial \tilde{u}_{i}}{\partial x_{j}}-\frac{\partial \tilde{u}_{j}}{\partial x_{i}}\right)=-\frac{\partial \tilde{p}^{*}}{\partial x_{i}}-\frac{\partial \tau_{i j}}{\partial x_{j}}+F_{i}
$$

where $\sim$ represents filtering. A spectral cutoff filter is used here as the implicit filter for the pseudospectral method applied in the present LES. $\tilde{u}_{i}$ is the filtered velocity and $F_{i}$ represents external forces. The convective terms are written in the rotational form for numerical kinetic energy conservation. The viscous diffusion term is neglected here because of the high Reynolds number of the flow and modeling of the surface layer near walls. The modified pressure, $\tilde{p}^{*}$, is defined as

$$
\tilde{p}^{*}=\tilde{p} / \rho+\tilde{u}_{k} \tilde{u}_{k} / 2+\left(\widetilde{u_{k} u_{k}}-\tilde{u}_{k} \tilde{u}_{k}\right) / 3 .
$$

$\tau_{i j}$ is the deviatoric (trace-free) part of the SGS stress, which is computed using an SGS model. Most SGS models are based on the "Smagorinsky model,"

$$
\tau_{i j}=-2\left(C_{s} \Delta\right)^{2}|\tilde{S}| \tilde{S}_{i j}
$$

where $\Delta$ is the filter size. $\tilde{S}_{i j}$ is the resolved strain rate, $\tilde{S}_{i j}=\left(\partial \tilde{u}_{i} / \partial x_{j}+\partial \tilde{u}_{i} / \partial x_{j}\right) / 2$, and $\tilde{S}=\sqrt{2 \tilde{S}_{i j} \tilde{S}_{i j}}$. The Smagorinsky coefficient, $C_{s}^{2}$, can be determined in terms of the dynamic procedure [14] involving a second filtering at scale $2 \Delta$ (denoted as ${ }^{\wedge}$ ), which, however, must be averaged over homogeneous flow directions to avoid numerical instability. Meneveau et al. have developed a Lagrangian procedure to obtain averages over fluid particle trajectories [22], that does not rely on specific flow conditions and makes the model particularly well suited for turbulent flows with complex configurations. The original dynamic procedure [14] assumes scale-invariance, i.e., $C_{s}^{2}$ is independent of filter scale. This assumption does not always hold, especially when the filter scale $\Delta$ approaches the integral scale, e.g., in the vicinity of a wall surface [21]. Porté-Agel et al. [29] proposed a scale-dependent dynamic model and applied it to atmospheric boundary-layer flows, but with averaging over homogeneous flow directions. Recently, Bou-Zeid et al. [4] have developed a scale-dependent Lagrangian 
dynamic model for complex turbulent flows, which is used in the present study. In this SGS model, an extra filtering at a larger scale, $4 \Delta$ (denoted as $\sim$ ), is employed to determine $\beta$, where $\beta=C_{s, 2 \Delta}^{2} / C_{s, \Delta}^{2}$ is a parameter that accounts for possible scale dependence of $C_{s, \Delta}^{2}$. $\beta$ is assumed to be scale invariant, i.e., $\beta=C_{s, 2 \Delta}^{2} / C_{s, \Delta}^{2}=C_{s, 4 \Delta}^{2} / C_{s, 2 \Delta}^{2}$. After computing $C_{s, 4 \Delta}^{2}$ and $C_{s, 2 \Delta}^{2}$ using the standard Lagrangian model, Bou-Zeid et al. [4] has shown that $C_{s, \Delta}^{2}$ at the grid scale can be expressed according to:

$$
C_{s, \Delta}^{2}=\frac{\mathcal{I}_{L M} / \mathcal{I}_{M M}}{\max \left(\frac{\mathcal{I}_{Q N} \mathcal{I}_{M M}}{\mathcal{I}_{N N} \mathcal{I}_{L M}}, 1 / 8\right)}
$$

where

$$
\mathcal{I}_{A B}=\int_{-\infty}^{t} A_{i j} B_{i j}\left[\mathbf{x}\left(t^{\prime}\right), t^{\prime}\right] W\left(t-t^{\prime}\right) d t^{\prime} .
$$

Here, $W(t)$ is an exponential weighting function, $W(t)=\mathrm{e}^{-t / T} / T$, and $T$ is a time scale, chosen as $T=1.5 \Delta\left(\mathcal{I}_{L M} \mathcal{I}_{M M}\right)^{-1 / 8}$ based on the direct numerical simulation data and selfconsistency [22]. $\mathbf{x}\left(t^{\prime}\right)$ is the location of fluid elements at a prior time $t^{\prime}$. A and $\mathbf{B}$ represent tensors $(\mathbf{L}, \mathbf{M})$ and $(\mathbf{N}, \mathbf{Q})$, respectively, computed as follows,

$$
\begin{aligned}
& L_{i j}=\widehat{\tilde{u}_{i} \tilde{u}_{j}}-\hat{\tilde{u}}_{i} \hat{\tilde{u}}_{j}, \\
& M_{i j}=2 \Delta^{2}\left[\overline{|\tilde{S}| \tilde{S}_{i j}}-4 \beta|\hat{\tilde{S}}| \hat{\tilde{S}}_{i j}\right] \text {, } \\
& Q_{i j}=\overbrace{\tilde{u}_{i} \tilde{u}_{j}}-\overbrace{\tilde{u}_{i}} \overbrace{\tilde{u}_{j}}, \\
& N_{i j}=2 \Delta^{2}[\overbrace{\tilde{S} \mid \tilde{S}_{i j}}-16 \beta|\overbrace{\tilde{S}}| \overbrace{\left.\tilde{S}_{i j}\right]}^{u_{j}}] .
\end{aligned}
$$

Equation 5 is equivalent to the following relaxation transport equation, that is more convenient for numerical discretization,

$$
\frac{\partial \mathcal{I}_{A B}}{\partial t}+\tilde{u}_{k} \frac{\partial \mathcal{I}_{A B}}{\partial x_{k}}=\frac{A_{i j} B_{i j}-\mathcal{I}_{A B}}{T} .
$$

For numerical expediency, the above transport equation is discretized with first-order accuracy in space and time (see [4] for detail).

The filtered Navier-Stokes equations (1) are discretized using a pseudospectral method in the horizontal directions and centered finite-difference method in the vertical direction [1,2]. The vertical velocity component, $w$, is staggered with horizontal components $u$ and $v$. In the pseudospectral method, the spatial derivatives are calculated in spectral space while the nonlinear convective terms are evaluated in physical space due to computational efficiency. The convective terms are de-aliased by padding and truncation using the $3 / 2$ rule [26]. The second-order Adams-Bashforth scheme is used for time advancement.

\subsection{Experimental setup of wind-tunnel model canopy}

Turbulent flows over a model canopy have been measured in the Corrsin Wind Tunnel at the Johns Hopkins University simultaneously by PIV and HA. Details on the experimental setup, procedures, and data analysis are provided in [47]. The test section in the wind tunnel has a length of $10 \mathrm{~m}$ and a cross section of $1.2 \times 0.91 \mathrm{~m}^{2}$. A schematic layout of the experimental setup is shown in Fig. 1a. The model canopy consists of $30 \mathrm{~cm}$ long wooden stalks with a diameter of $3.2 \mathrm{~mm}$. They are placed in $5 \mathrm{~cm}$ thick styrofoam in a staggered configuration 


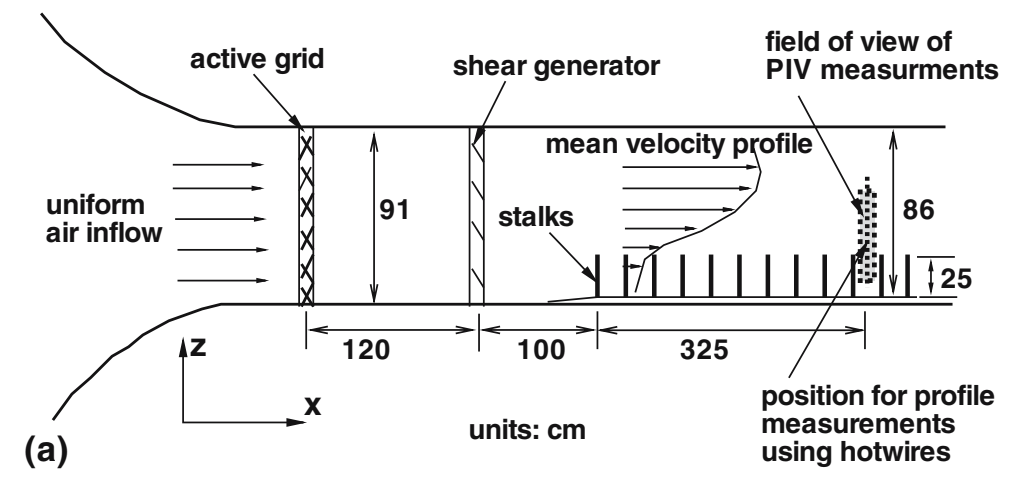

(b)

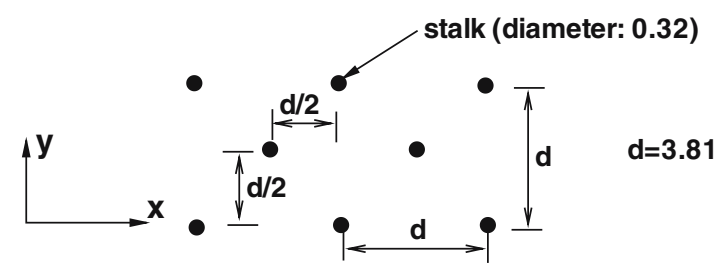

Fig. 1 Schematic of wind-tunnel experiment setup (not to scale). (a) Layout in the vertical plane. (b) Distribution of stalks in the horizontal plane. All units are in centimeter

(see Fig. 1b). The height of the model canopy is $h=25 \mathrm{~cm}$. The stalks fill in the spanwise direction in the whole test section over a streamwise distance of $4.6 \mathrm{~m}$. The turbulence level is enhanced by an active grid [18] placed $1.2 \mathrm{~m}$ upstream of a shear generator. The shear generator, located $1 \mathrm{~m}$ upstream of the front of the model canopy, is designed to create the same normalized mean shear profile as obtained in field experiments described in [49]. The PIV system consists of one cross-correlation CCD camera ( $2 k \times 2 k$, Kodak ES4.0, 8 bit), and an Nd:Yag laser (120 mJ/pulse, New-Wave Research). A vertical light sheet is generated at the centerline of the wind tunnel. The field view is $4.8 \times 4.8 \mathrm{~cm}^{2}$ in the middle of two rows of the stalks, located $3.25 \mathrm{~m}$ downstream of the front stalks. The measurements are performed between $z / h=0.81$ and 2.13. For each elevation, 2360 vector maps are acquired. In the HA measurements, a custom-made miniature I-type HA probe is used. It contains one $2.5 \mu \mathrm{m}$ platinum-coated tungsten wire, which is copper-plated and soldered to the I-wire prong ends and etched, yielding an active length-to-diameter ratio of about 200. The HA operates in a constant temperature anemometry (CTA) mode by TSI IFA300 unit. The overheat ratio of the hot-wires is about 1.6. The signals are sampled at $40 \mathrm{kHz}$, low-pass filtered at a frequency of $20 \mathrm{kHz}$ and digitized with a 12 bit Simultaneous Sample and Hold A/D converter (United Electronic Industries, WIN-30DS). Sampling time is 90 s, so the total number of data points per channel for each measurement location is $3.6 \times 10^{6}$. The data are sampled in the elevation range between $z / h=0.425$ and 2.4 .

\subsection{Numerical simulation of wind-tunnel model canopy}

At the measurement location, streamwise changes in the mean velocity profiles have been found to be very small. Therefore, the LES assumes that the flow is fully developed and uses the periodic boundary conditions in the streamwise direction. Since the measurements have been performed in the middle of the wind tunnel, where the effects of lateral walls 
are minimal, spanwise periodic boundary conditions are used in the LES. In the vertical direction, the computational domain matches the full wind tunnel height. The bottom and top boundaries are treated as no-slip walls, and are modeled by the law-of-the-wall, see [4] for detailed implementation. The flow is driven by a mean pressure gradient.

The computational box in the LES uses the actual wind tunnel sizes, with length scales $L_{x}: L_{y}: L_{z}=3.66 h: 1.83 h: 3.4 h$, where $h$ is the stalk height and $x, y$, and $z$ are streamwise, spanwise, and vertical directions, respectively. The grid resolution is $N_{x}: N_{y}: N_{z}=96: 48$ : 86. These length scales have been chosen such that the distance between two neighboring streamwise (or spanwise) stalks is resolved by four uniform grid cells. The integral scale of the flow is about $2-3 \mathrm{~h} \mathrm{[49].} \mathrm{Our} \mathrm{previous} \mathrm{study} \mathrm{shows} \mathrm{that} \mathrm{this} \mathrm{streamwise} \mathrm{size} \mathrm{is} \mathrm{adequate}$ for predicting most energetic turbulent eddies in this flow [46]. In the vertical direction, a stalk is resolved by 25 uniform grid cells in the vertical direction. The stalks in the wind tunnel act as drag forces in the flow field. In the present LES, the stalk drag force per unit mass, $\mathbf{d}$, is calculated based on a cylinder drag formula,

$$
\mathrm{d}_{i}=-\frac{1}{2} C_{0} \frac{D}{\mathrm{~d} x \mathrm{~d} y}\left|\tilde{\mathbf{u}}_{0}\right| \tilde{u}_{0, i}
$$

where $D$ is the diameter of the model stalk, and $\mathrm{d} x$ and $\mathrm{d} y$ are grid spacings in the streamwise and spanwise directions, respectively. The parameter $C_{0}$ is the cylinder drag coefficient. For high Reynolds number flows and for isolated cylinders, the drag coefficient is near 1.0 [32]. In the present application the cylinders are attached on one side to the ground, and there may be some interference effects among cylinders as they are placed in an array. The specified value of $C_{0}$ directly controls the momentum exchange among the canopy and the wind, and results are expected to be sensitive to the value specified. However, since the length-diameter aspect ratio (about 75:1) and the spanwise spacing between sticks $(D / d=12)$ are relatively large, deviation from the baseline case $C_{0}=1.0$ are not expected to be very significant. As a matter of fact, based on Jacob's correlation for an array of tubes [20], drag coefficient in present spanwise spacing of sticks is within 5\% deviation from 1.0. Therefore, the value $C_{0}=1.0$ is used in the simulations. In Eq. 8, $\tilde{u}_{0, i}$ are the upstream velocity components, calculated at a distance $L$ upstream of the stalk points (see Fig. 2). $L$ is set to $3 D$ in this study. $\left|\tilde{\mathbf{u}}_{0}\right|$ is the magnitude of the upstream velocity, equal to $\sqrt{\tilde{u}_{0, i} \tilde{u}_{0, i}}$. The upstream velocity $\tilde{u}_{0, i}$ is calculated by a trilinear interpolation in terms of the corner point velocity in the grid cell containing the upstream point $P(x, y, z)$,

$$
\begin{aligned}
\tilde{u}_{0, i}(x, y, z)= & \left(1-s_{1}\right)\left(1-s_{2}\right)\left(1-s_{3}\right) \tilde{u}_{i}^{1}+s_{1}\left(1-s_{2}\right)\left(1-s_{3}\right) \tilde{u}_{i}^{2}+s_{1} s_{2}\left(1-s_{3}\right) \tilde{u}_{i}^{3} \\
& +\left(1-s_{1}\right) s_{2}\left(1-s_{3}\right) \tilde{u}_{i}^{4}+\left(1-s_{1}\right)\left(1-s_{2}\right) s_{3} \tilde{u}_{i}^{5}+s_{1}\left(1-s_{2}\right) s_{3} \tilde{u}_{i}^{6} \\
& +s_{1} s_{2} s_{3} \tilde{u}_{i}^{7}+\left(1-s_{1}\right) s_{2} s_{3} \tilde{u}_{i}^{8}
\end{aligned}
$$

where $s_{1}=(x-x 1) /(x 2-x 1), s_{2}=(y-y 1) /(y 2-y 1)$, and $s_{3}=(z-z 1) /(z 2-z 1)$. $\tilde{u}_{i}^{k}(k=1,2, \ldots, 8)$ is the velocity at the corner points of the interpolation box.

\section{Results and discussion}

The predicted turbulence statistics, energy spectra, and TKE budgets by the LES are compared with the PIV and HA experimental data where available. Only two-dimensional (streamwise and vertical) data were acquired with the PIV measurements and one-dimensional (streamwise) data with the HA measurements. The superscript ', e.g., in $u^{\prime} w^{\prime}$, denotes temporal fluctuation, i.e., deviation from local time-averaged mean value (the latter is denoted as 


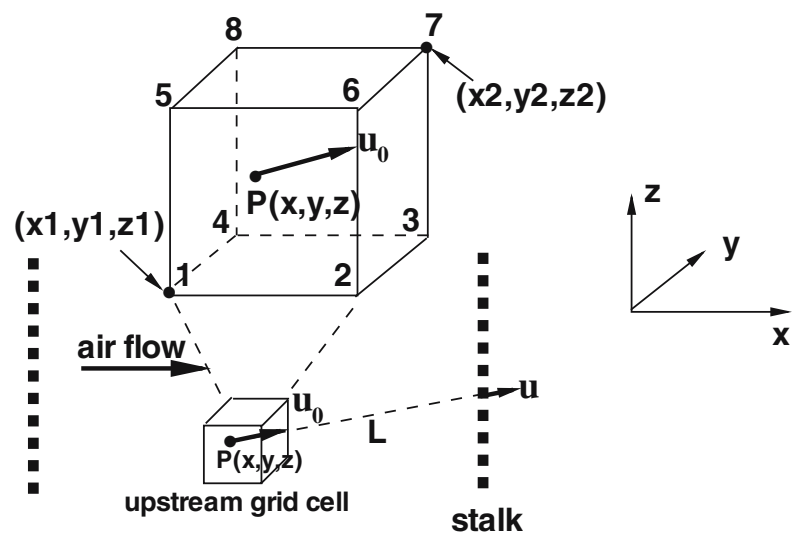

Fig. 2 Schematic for the calculation of upstream velocity $\tilde{u}_{0}$

overbar). The superscript " , e.g., in $u^{\prime \prime}$, denotes spatial variation, i.e., deviation from the horizontally-averaged mean value (the latter is denoted as \langle\rangle ). All turbulence statistics predicted by the LES are calculated in terms of temporal fluctuations. The resulting three dimensional statistics are then averaged over horizontal planes to represent the overall effects of the model canopy.

\subsection{Mean velocity profile and instantaneous flow field}

In Fig. 3, the mean streamwise velocity profiles from the LES, the PIV, and the HA are compared. Here $\langle\overline{\tilde{u}}(z)\rangle$ is temporally and spatially averaged for the LES and PIV data, while the HA data are only averaged in time. There is, in general, very good agreement between the experimental PIV and HA profiles. The PIV and HA profiles are nearly linear above the stalks due to the linear shear velocity profile in front of the model canopy generated by the shear generator. Some discrepancy with the LES mean velocity profile is observed, whose periodic boundary condition yields a profile that is not quite linear above the canopy. Also the velocity decreases sharply inside the model canopy, where the LES prediction of $\langle\overline{\tilde{u}}(z)\rangle$ falls somewhat below the experimental data. The LES prediction of mean velocity also shows the expected rapid decrease when the top boundary (the wind-tunnel top wall) is approached. The PIV and HA data measurements do not extend to the top of the wind tunnel so no experimental data are available there. All the profiles are inflected at the stalk top, implying that hydrodynamic instability can exist at the top of the model canopy. An analogy between canopy turbulence and mixing layer turbulence may be used to define a shear length scale [10], according to

$$
L_{s}=\frac{\langle\overline{\tilde{u}}(h)\rangle}{(\partial\langle\tilde{\tilde{u}}(z)\rangle / \partial z)_{z=h}},
$$

It is equal to $0.3 \mathrm{~h}$ from the LES profile.

A sample of an instantaneous velocity distribution in a vertical plane $\left(y=L_{y} / 2\right)$ obtained from the LES is given in Fig. 4a. The velocity vectors are shown at every other point for clarity. The momentum transfer between air and the model canopy is seen clearly, since velocity is significantly damped inside the model canopy due to the stalk drag. To visualize the flow structures around the top of the model canopy, the velocity at the stalk top (averaged in the 


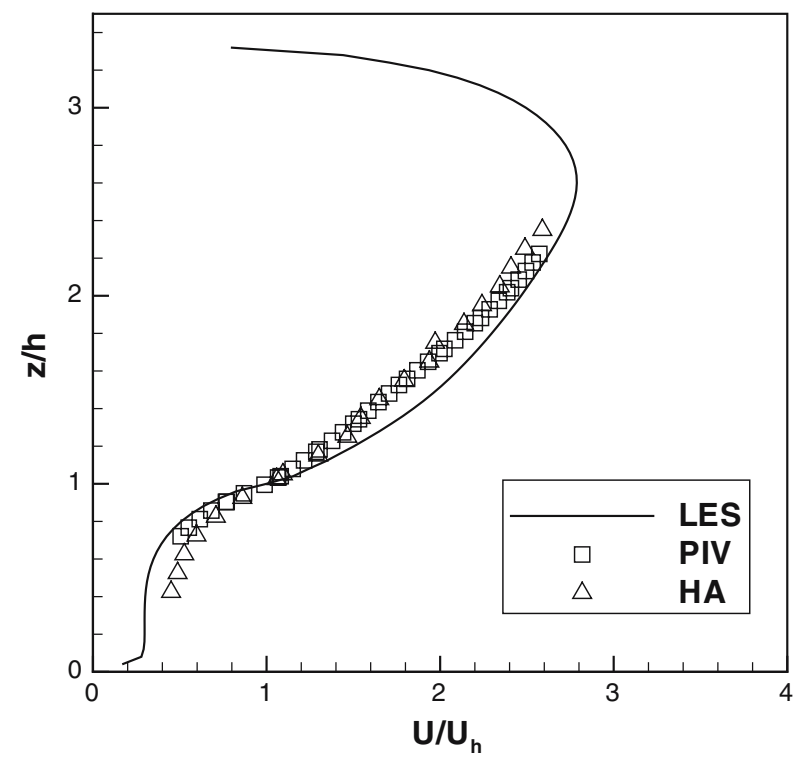

Fig. 3 Mean streamwise velocity profiles from simulations and measurements. large eddy simulation (LES); particle image velocimetry (PIV); hotwire anemometer (HA)

streamwise direction) is subtracted from the streamwise velocity component in Fig. 4b. The vortices around the stalk top are easily identified. Their size is of the same order as $L_{S}$, and they result from Kelvin-Helmholtz-like instability around the top of the stalks [10]. These vortices dominate the flow there and thus play a significant role in the momentum and energy transfer between the air and the model canopy as discussed later in more detail.

\subsection{Energy spectra}

Figure 5 shows the one-dimensional energy spectra of $u^{\prime \prime}, E_{u u}$, from the LES, the PIV, and the $\mathrm{HA}$, with respect to the streamwise wavenumber, $k_{x}$. Results are shown at the four elevations from inside the stalks to above the stalks, $z / h=0.87,1,1.4$, and 2 . The data are normalized by the stalk height $h$ and the friction velocity at the top of the stalks $u_{*}=\left.\sqrt{\left\langle-\overline{u^{\prime} w^{\prime}}\right\rangle}\right|_{z=h}$. The spectra are averaged over the spanwise direction and in time in the LES. The temporal HA frequency is converted into spatial wavenumber using Taylor's Hypothesis. The energy spectra profiles from the LES, the PIV, and the HA nearly collapse at all the four elevations. The small peak visible in the LES spectrum at $k_{x} h=40$ for $z / h=0.87$ and 1.0 results from the canopy drag imposed in the numerical model with periodicity of $4 d x$. The HA spectra have the longest wavenumber span, over three decades. The LES and PIV data each span about one and half decades. Compared to the experimental data, the LES results show a faster roll-off rate between the filter scale and the stalk spacing length. At these scales, consistent with the arguments of Finnigan [10], energy is not only cascaded in the inertial range, but is also directly extracted by the interactions with the canopy.

Next, one-dimensional energy spectra of $w^{\prime \prime}, E_{w w}$, at the four elevations from the LES and the PIV are presented in Fig. 6. The combination of the LES and the PIV spans three decades of the wavenumber space, and the spectra of the LES and the PIV results agree well within the common wavenumber range. The faster roll-off rate of $E_{u u}$ than $E_{w w}$ was 

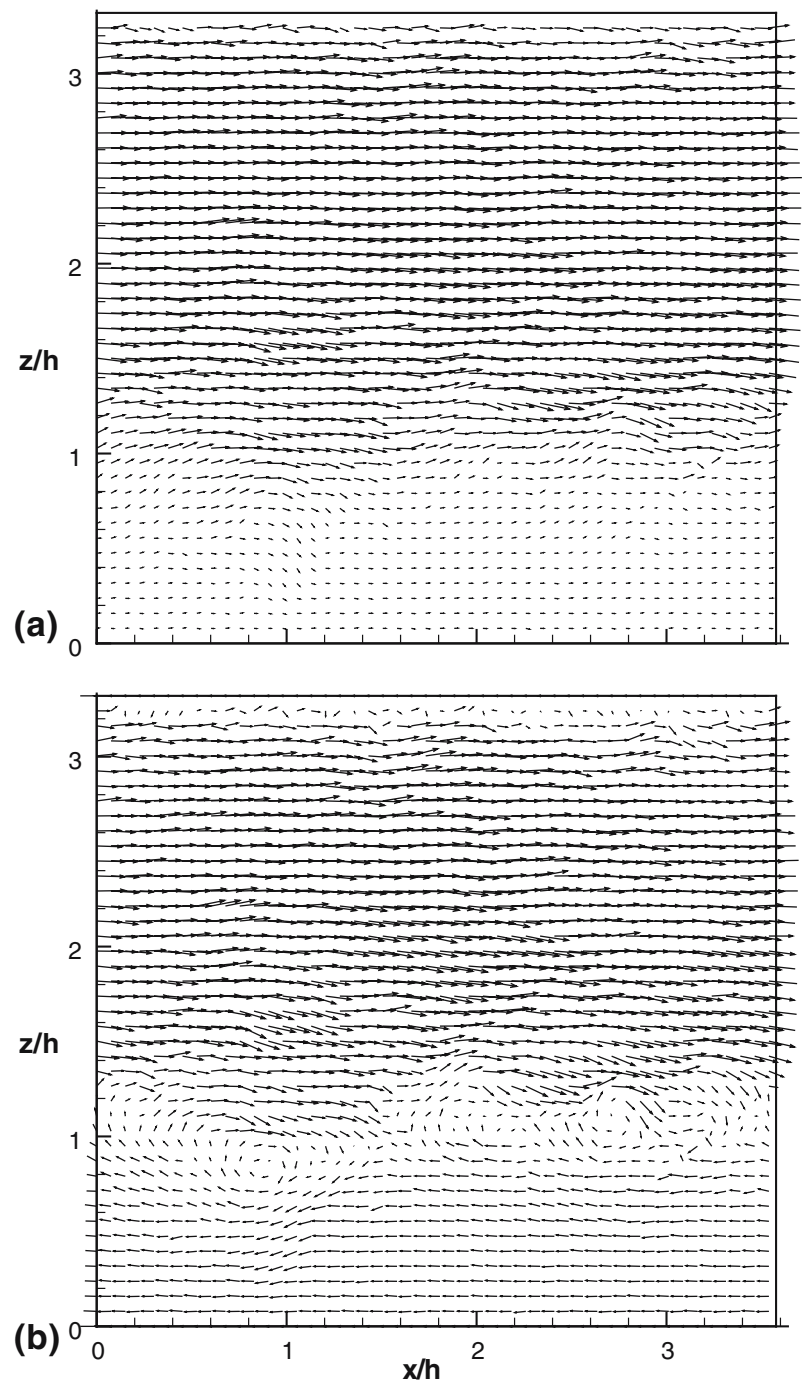

Fig. 4 An instantaneous velocity field from LES in a vertical plane. (a) Vector of $\tilde{u}$ and $\tilde{w}$; (b) vector of $\tilde{u}-\langle\tilde{u}(h)\rangle$ and $\tilde{w}$, where $\langle\tilde{u}(h)\rangle$ is the velocity at the canopy top (averaged in the streamwise direction)

also observed in [40] for forest canopies. The energy spectra $E_{u u}, E_{v v}$, and $E_{w w}$ predicted from the LES at the four elevations are plotted together in Fig. 7. Consistent with small-scale isotropy, $E_{v v}$ has a good collapse with $4 / 3 E_{u u}$ at all elevations. $E_{w w}$, however, does not collapse with $4 / 3 E_{u u}$, indicating a deviation from local isotropy in this flow, consistent with prior observations [10]. The peaks at $k_{x} h=40$ for the cases at and below the stalk top result from the periodicity of the imposed stalk drag in the numerical simulation, as mentioned above.

\subsection{Root-mean-square velocity, shear stress, and correlation coefficient}

The root-mean-square (rms) velocity profiles, $u_{\mathrm{rms}}, v_{\mathrm{rms}}$, and $w_{\mathrm{rms}}$, from the LES and both sets of measurements, normalized by $u_{*}$, are shown in Fig. 8. There is quite good agreement 

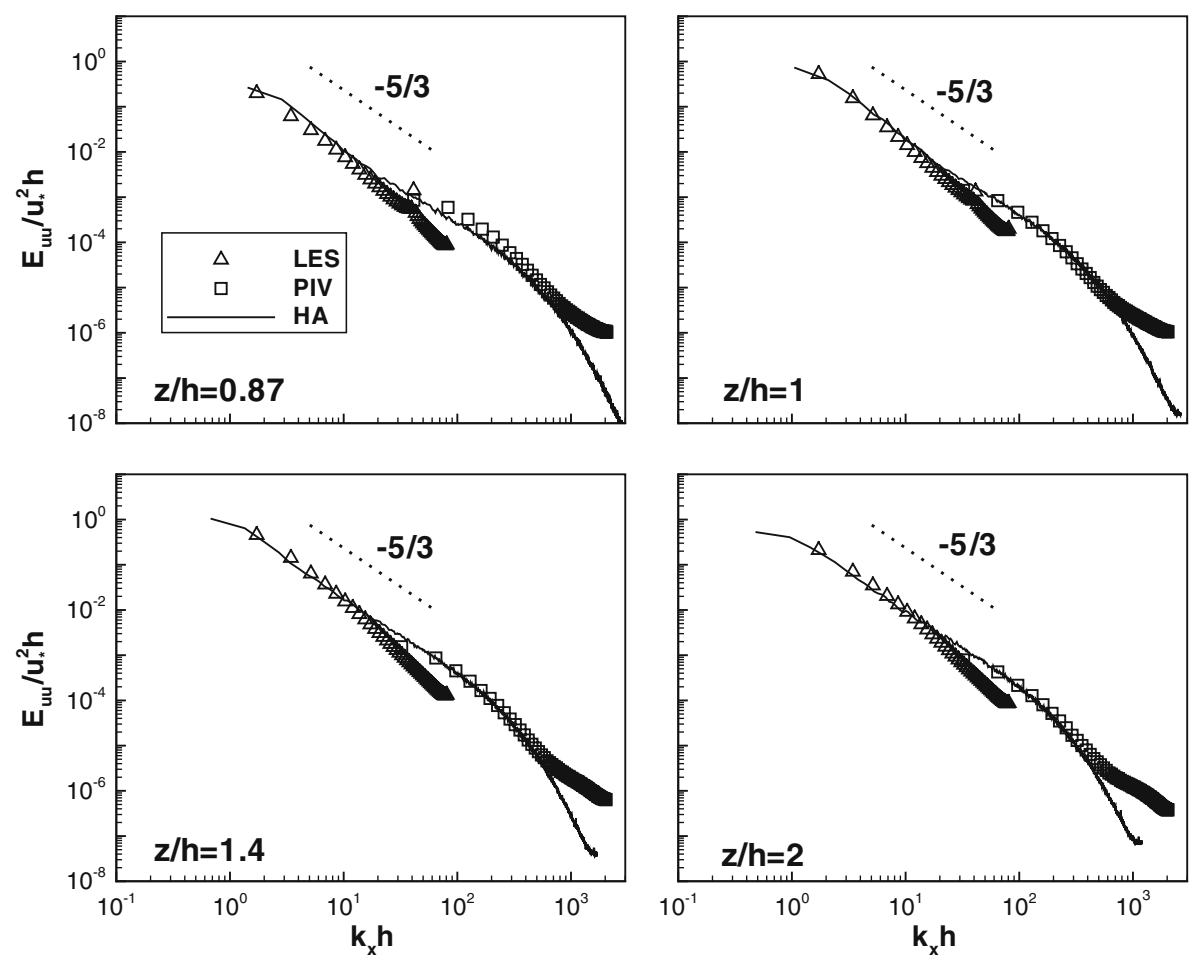

Fig. 5 Energy spectra $E_{u u}$ at four elevations

in $u_{\mathrm{rms}}$ and $w_{\text {rms }}$ obtained from the LES and the PIV and HA measurements. The fluctuations decrease quickly within the stalks. The three rms profiles obtained from the LES also show peaks near the upper wall where the strong near-wall turbulence production occurs. The normalized $u_{\mathrm{rms}}$ and $w_{\mathrm{rms}}$ are around 1.65 and 1.15 , respectively, which are close to the values of 1.8 and 1.2 observed in LES of the corn field [46] and 1.8 and 1.1 observed in PIV measurements of the same corn field [41]. Similarly, [40] showed respective values of 1.7 and 1.05 for LES of a forest canopy. Contrary to what is normally observed in atmospheric turbulence, $v_{\text {rms }}$ is slightly smaller than $w_{\text {rms }}$ at all levels of the wind tunnel except near the top and bottom walls. $u_{\text {rms }} /\langle\overline{\tilde{u}}(z)\rangle$ and $w_{\text {rms }} /\langle\overline{\tilde{u}}(z)\rangle$ are used to measure the local turbulence intensity (see Fig. 9). There is very good agreement between the LES and the experimental measurements above the stalks, but some departure within the stalks. There is a large local turbulence intensity immediately below the top of the stalks. Peaks are also observed near the upper wall. Immediately below the canopy top, $u_{\mathrm{rms}}$ decreases rapidly, and the HA data show smaller $u_{\text {rms }}$ and larger $\langle\overline{\tilde{u}}(z)\rangle$ than the PIV data, resulting in the relatively large difference in turbulence intensity between the PIV and HA data in Fig. 9.

The vertical profiles of the Reynolds shear stress (including the mean SGS stress), $-\left\langle\overline{u^{\prime} w^{\prime}}\right\rangle$ $-\left\langle\tau_{\text {sgs }}\right\rangle$, also called the momentum flux, normalized by $u_{*}^{2}$, are given in Fig. 10 . The agreement between the LES results and the PIV data is good although note that both are normalized to agree at $z / h=1$. The momentum flux deceases sharply below the top of the stalks, similar to the rms velocity in Fig. 8. In the lower layer of the model canopy, the momentum flux nearly vanishes, indicating that most of the momentum transported from the top of the stalks is absorbed by the upper layer of the model canopy. On the other hand, the rms velocities are 

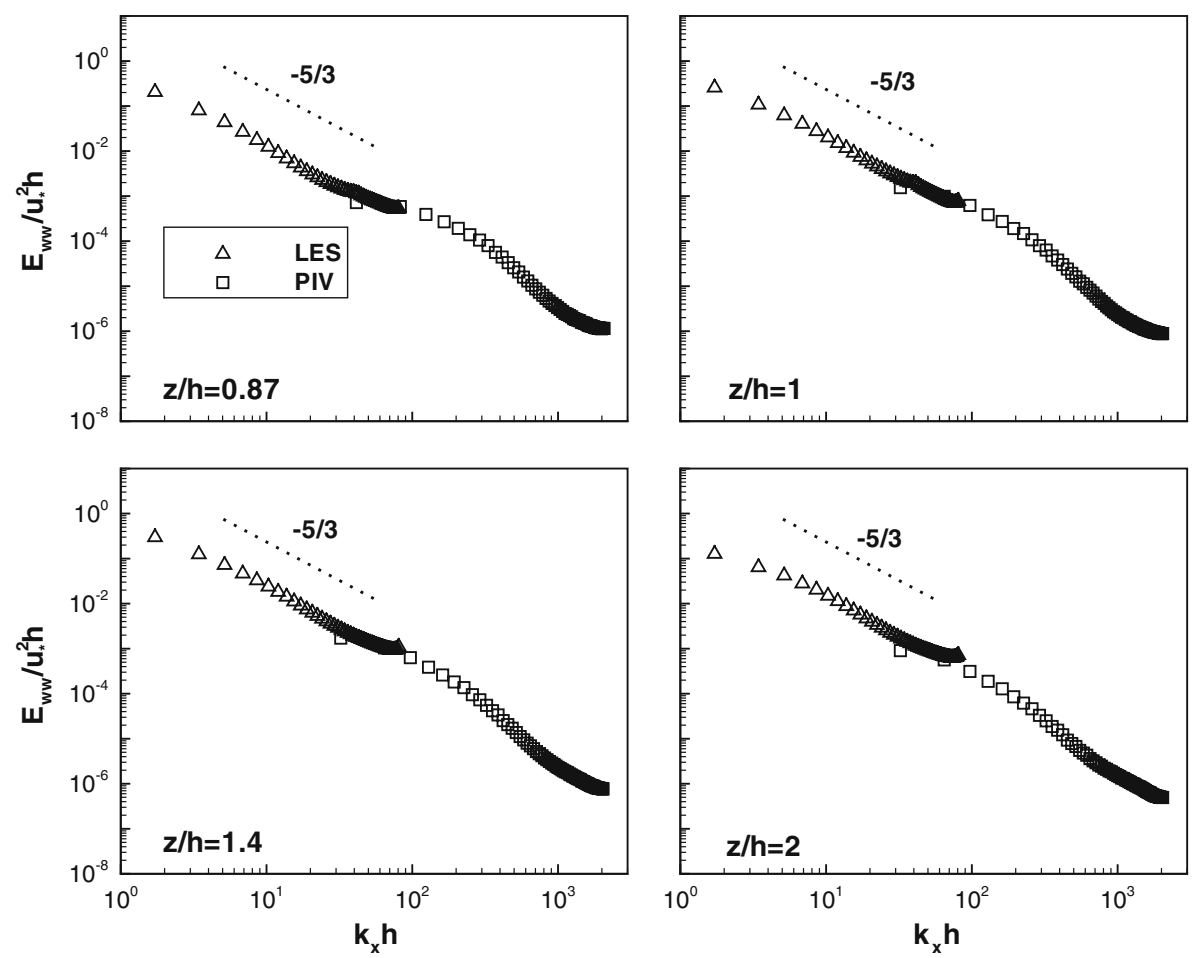

Fig. 6 Energy spectra $E_{w w}$ at four elevations

finite in this lower layer and the streamwise component is larger than the other two components. Similar flow behavior was observed in the corn canopy with even larger streamwise fluctuations [46]. This is usually called inactive "sloshing" motions which contribute little in the transfer of momentum [10], as will be further discussed in the subsequent section. Above the stalks, the momentum flux shows a linear profile, as in a turbulent channel flow, suggesting that the main effect of the model canopy on the flow above it is to modulate the friction velocity at the top of the stalks. The momentum flux crosses zero at $z / h=2.7$, very close to where the rms velocities attain their minimum values (see Fig. 8) and the mean velocity attains its maximum value as well (see Fig. 3).

The correlation coefficient, $R_{u w}=\left\langle\overline{u^{\prime} w^{\prime}}\right\rangle /\left(u_{\mathrm{rms}} w_{\mathrm{rms}}\right)$, is plotted in Fig. 11. $R_{u w}$ describes the efficiency of momentum transport. There is excellent agreement between the LES and the PIV. Both the LES and PIV data show a peak at the top of the stalks, indicating that this is the location where turbulence is the most efficient in transporting momentum. Both the LES and the PIV show a peak value of -0.6 , consistent with reported values in the literature [10]. The same value of the peak was also observed in [40] for the forest canopy, but in their case the peak occured at $z / h=0.87$, possibly due to differences in the imposed drag distribution.

\subsection{Velocity skewness and flatness}

In Fig. 12, the velocity skewness of streamwise and vertical components are presented (e.g., $\left.S_{u}=\left\langle\overline{u^{\prime 3}}\right\rangle / u_{\mathrm{rms}}^{3}\right)$. There is good agreement between the LES and the experimental data above the stalks, especially for $S_{w}$, but some deviations inside the stalks and well above the canopy 

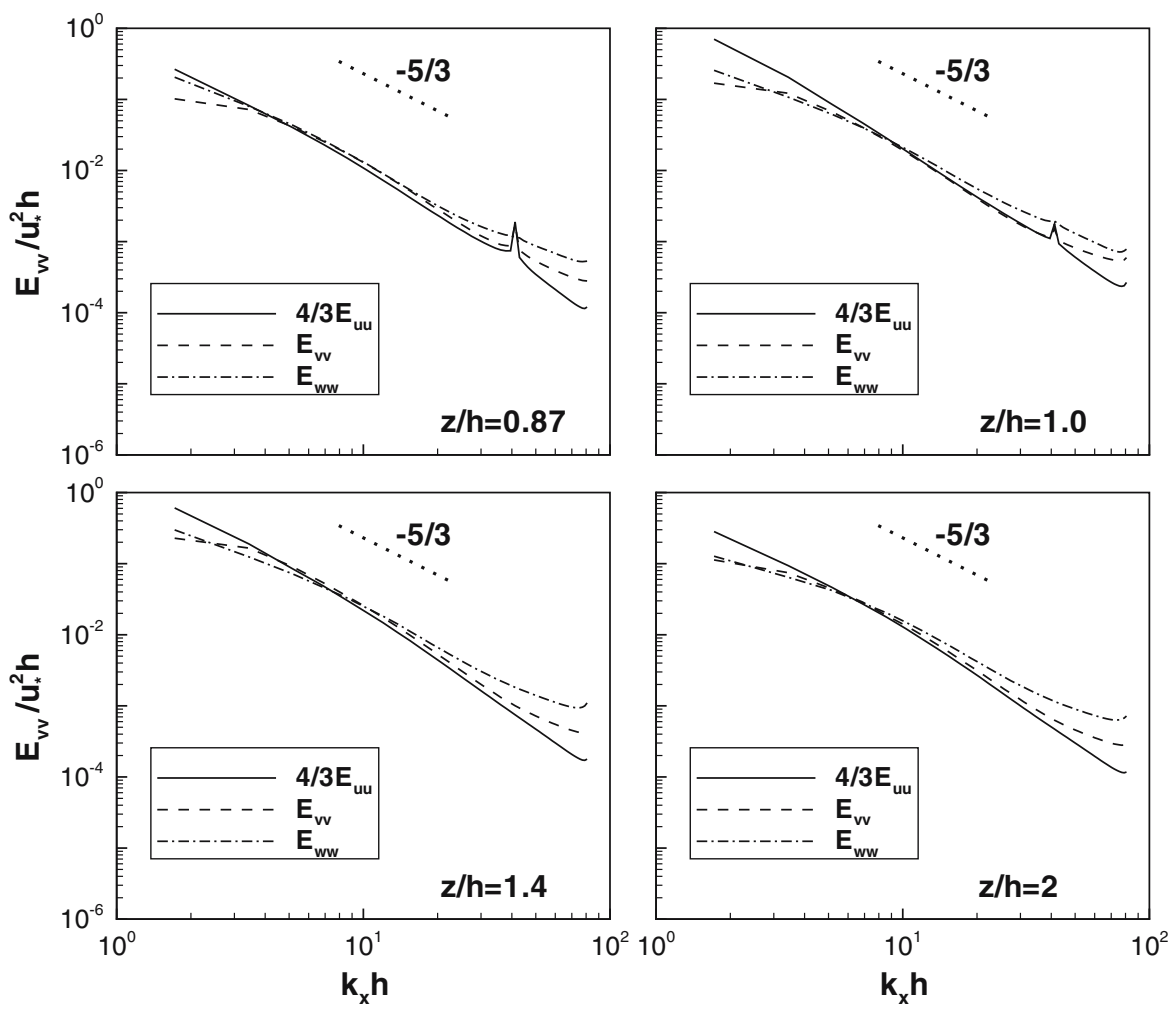

Fig. 7 Superimposed Energy spectra of $4 / 3 E_{u u}, E_{v v}$, and $E_{w w}$ at four elevations

for $S_{u}$. Similar deviations were reported in [40] in their forest canopy LES. In general, using smaller grid sizes yields bigger velocity skewness as shown in [25]. The discrepancy inside the canopy suggests that the skewness is dominated by motions at scales smaller than the grid size used in the LES. The same reasoning may explain the difference between the PIV and HA data.

The fourth order moment, velocity flatness (or kurtosis), is shown in Fig. 13 for both streamwise and vertical components, e.g., $F_{u}=\left\langle\overline{u^{\prime 4}}\right\rangle / u_{\mathrm{rms}}^{4}$. The velocity flatness is a good indicator of turbulence intermittency. There is quite good agreement among the numerical simulation and the experiments, except that inside the stalks $F_{u}$ obtained from the HA data is larger than the LES and PIV data. Note that both the LES and PIV do not measure down to the smallest scales. The higher flatness obtained from the HA data suggests that it is associated with very small scale motions not well resolved by the LES and PIV. Inside the stalks, the flatness profiles are somewhat similar to those of the skewness: the maximum for the streamwise component is immediately below the top of the stalks, and the maximum for the vertical component is located next to the bottom wall, where fewer downward eddies are able to penetrate deep into the model canopy. The profiles above the stalks, however, are quite different from those of the skewness. The $F_{u}$ from the LES reaches a maximum value of 10.6 at $z / h=2.7$, and at $z / h=2.6, F_{w}$ shows a maximum value of 4.7. In the layer above the stalks, $1<z / h<1.6$, both $F_{u}$ and $F_{w}$ are close to the Gaussian value of 3 , indicative of less intermittent turbulence motions within this air layer. The large values of the velocity 


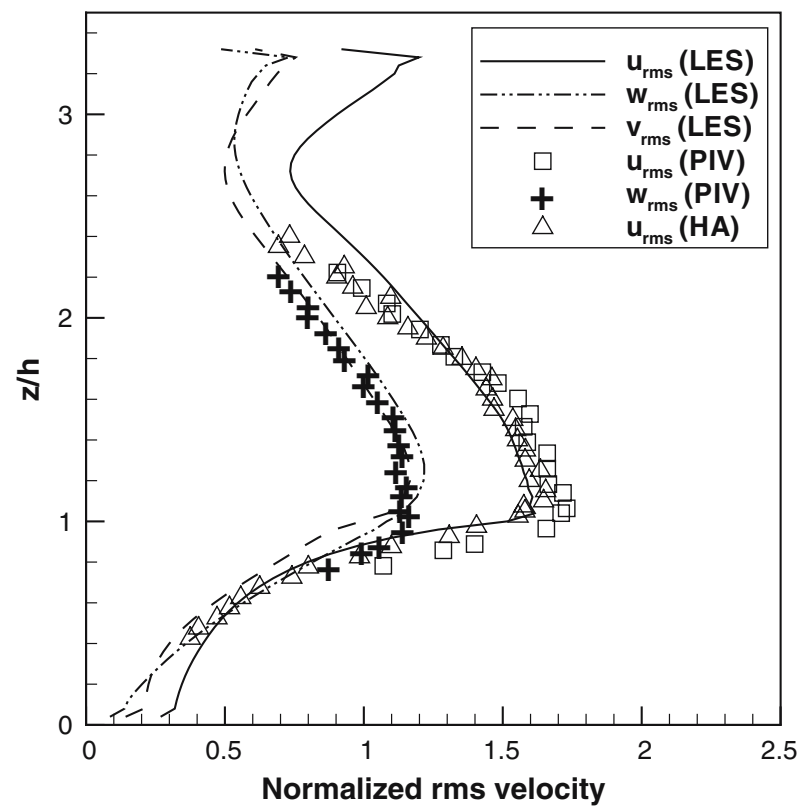

Fig. 8 RMS velocities, normalized by $u_{*}$ (the friction velocity at the canopy top)
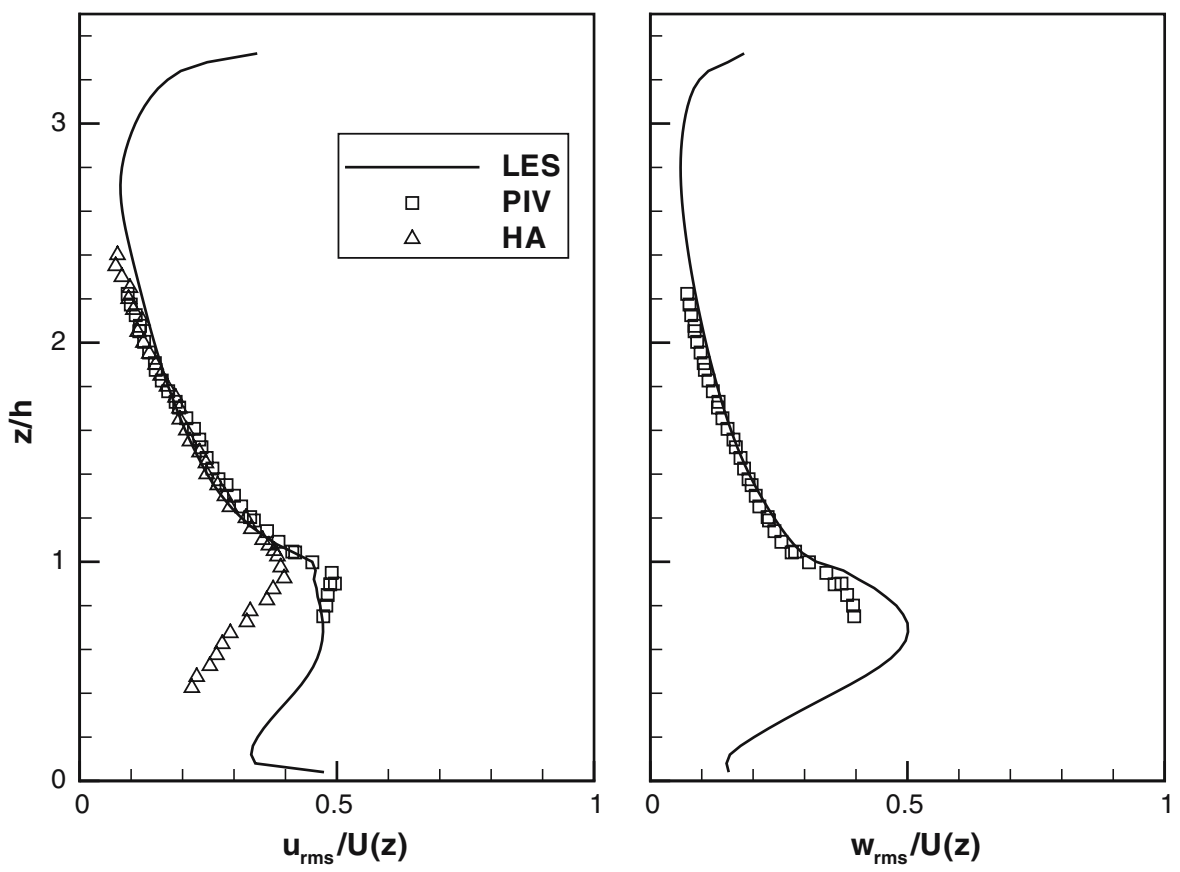

Fig. 9 Local turbulence intensity profile 


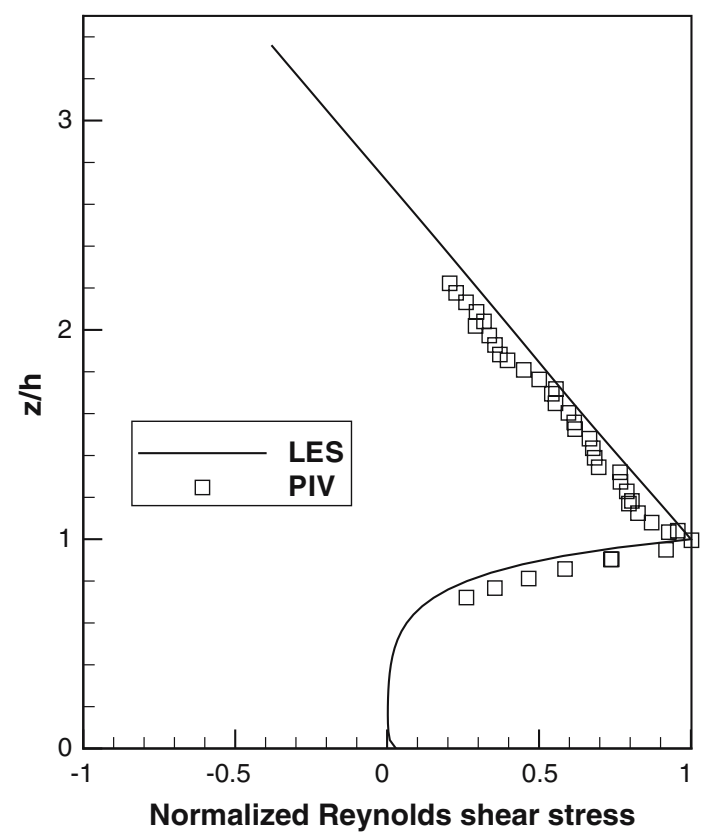

Fig. 10 Mean shear stress (sum of the resolved-scale and SGS components)

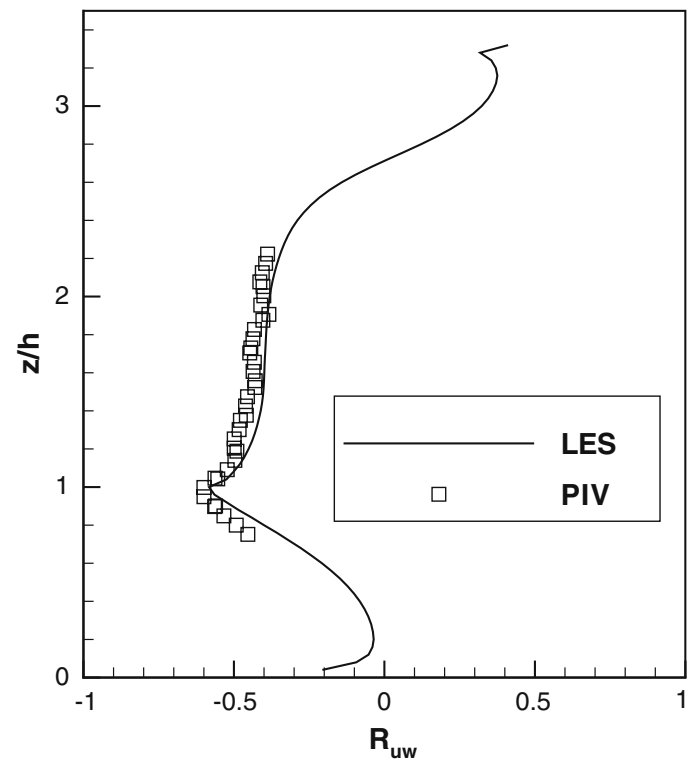

Fig. 11 Correlation coefficient of $\tilde{u}^{\prime}$ and $\tilde{w}^{\prime}$ 

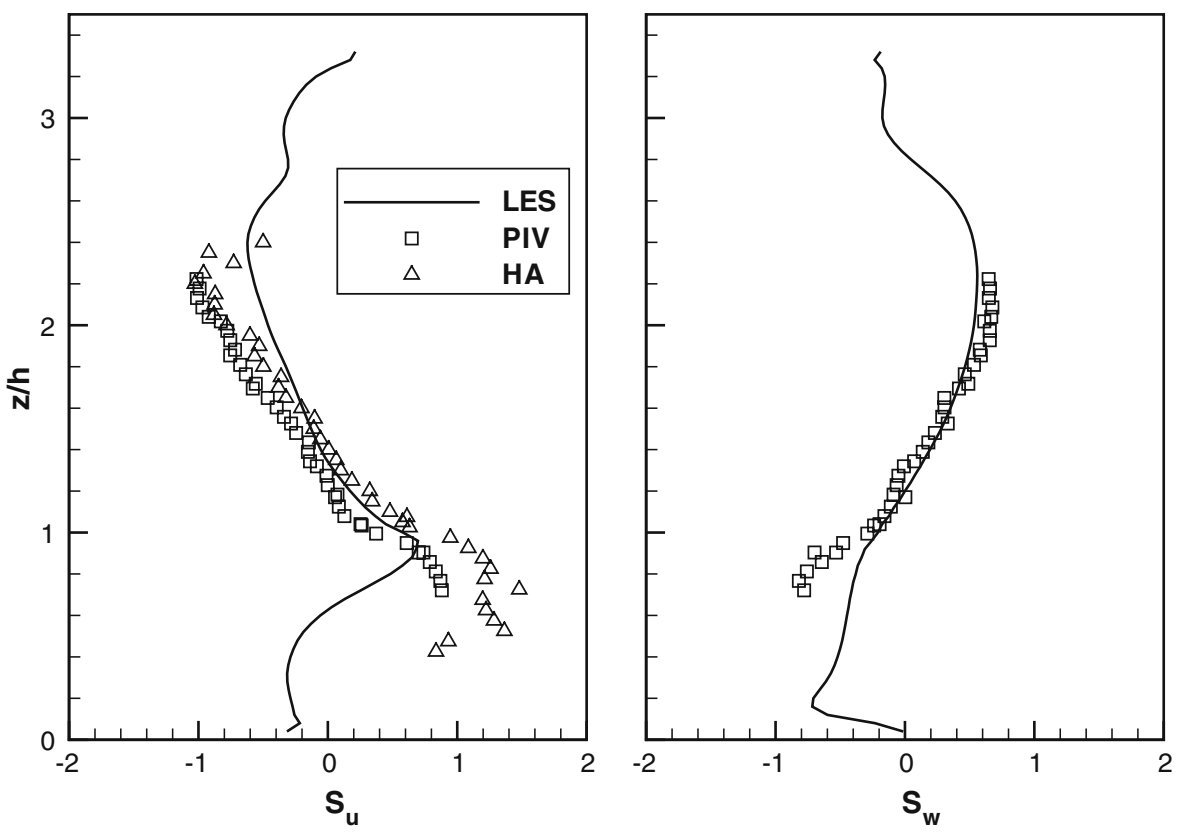

Fig. 12 Velocity skewness of streamwise and vertical velocity components

flatness within $2<z / h<3$, where the skewness is also far from zero and the rms velocity has a minimum (Fig. 8), indicates that the turbulence is highly intermittent in this region.

\subsection{Turbulent kinetic energy budget}

An analysis of TKE budgets is useful as it provides information on the relative significance of various physical processes that govern turbulent motions. The resolved-scale TKE budget equation is

$$
\frac{\partial \bar{K}}{\partial t}+\overline{\tilde{u}}_{i} \frac{\partial \bar{K}}{\partial x_{i}}=-\overline{u_{i}^{\prime} u_{j}^{\prime}} \frac{\partial \overline{\tilde{u}}_{j}}{\partial x_{i}}-\frac{\partial \overline{K u_{i}^{\prime}}}{\partial x_{i}}-\frac{\partial \overline{p^{\prime} u_{i}^{\prime}}}{\partial x_{i}}-\frac{\partial \overline{\tau_{i j}^{\prime} u_{j}^{\prime}}}{\partial x_{i}}+\overline{d_{i}^{\prime} u_{i}^{\prime}}+\overline{\tau_{i j}^{\prime} \tilde{S}_{i j}^{\prime}}
$$

$K$ is the resolved-scale turbulent kinetic energy, $\left(\tilde{u}^{\prime 2}+\tilde{v}^{\prime 2}+\tilde{w}^{\prime 2}\right) / 2$, and both $i$ and $j$ represent $x, y$, and $z$ components. In previous works on TKE budgets analysis of canopy flows, the time-averaged mean quantities are further decomposed into a volume-averaged quantity and a departure from the volume-averaged quantity $[8,10,30,39]$. As a result, extra terms associated with the departures from volumetric averaging appear in the TKE budget equation presented, e.g., wake production (a supplement to $P_{S}$ ), dispersive transport (a supplement to $T_{t}$ ), etc. In this work we opt to apply a horizontal averaging operation $(\langle\rangle)$ on each term of Eq. 11 without further spatial decompositions. This simplifies the subsequent comparisons with experimental data. From horizontal homogeneity the resulting terms containing horizontal derivatives vanish, and we can simplify Eq. 11 as follows:

$$
0=-\left\langle\overline{u^{\prime} w^{\prime}} \frac{\partial \overline{\tilde{u}}}{\partial z}\right\rangle-\left\langle\frac{\partial \overline{K w^{\prime}}}{\partial z}\right\rangle-\left\langle\frac{\partial \overline{p^{\prime} w^{\prime}}}{\partial z}\right\rangle-\left\langle\frac{\partial \overline{\tau_{i z}^{\prime} u_{i}^{\prime}}}{\partial z}\right\rangle+\left\langle\overline{d_{i}^{\prime} u_{i}^{\prime}}\right\rangle+\left\langle\overline{\tau_{i j}^{\prime} \tilde{S}_{i j}^{\prime}}\right\rangle
$$



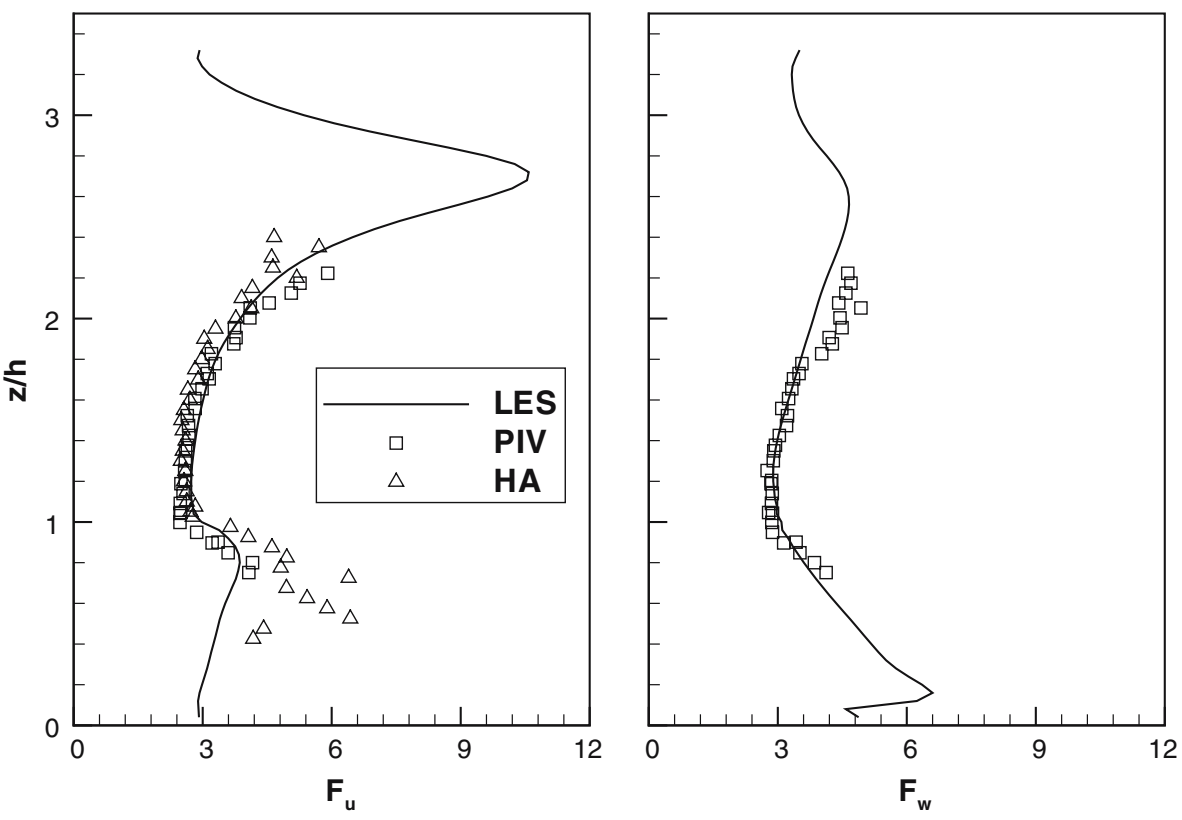

Fig. 13 Velocity flatness (kurtosis) of streamwise and vertical velocity components

The terms in Eq. 12 are referred to as $P_{S}\left(-\left\langle\overline{u^{\prime} w^{\prime}} \partial \overline{\tilde{u}} / \partial z\right\rangle\right.$, shear production), $T_{t}\left(-\left\langle\partial \overline{K w^{\prime}} / \partial z\right\rangle\right.$, turbulent transport), $T_{p}\left(-\left\langle\partial \overline{p^{\prime} w^{\prime}} / \partial z\right\rangle\right.$, pressure transport), $T_{\mathrm{sgs}}\left(-\left\langle\partial \overline{\tau_{i z}^{\prime} u_{i}^{\prime}} / \partial z\right\rangle\right.$, SGS transport $), W_{d}\left(\left\langle\overline{d_{i}^{\prime} u_{i}^{\prime}}\right\rangle\right.$, rate of work against the canopy drag), and $-\epsilon_{f}\left(\left\langle\overline{\tau_{i j}^{\prime} \tilde{S}_{i j}^{\prime}}\right\rangle\right.$, dissipation $) . P_{s}$ represents the conversion of mean flow kinetic energy to the resolved-scale TKE. $T_{t}, T_{p}$, and $T_{\text {sgs }}$ represent the vertical transport of the resolved-scale TKE by fluctuations of vertical velocity, pressure, and SGS stresses, respectively. $-\epsilon_{f}$ represents the energy transfer from the resolved-scale TKE to the SGS. A further decomposition of $\epsilon_{f}$ reads,

$$
\overline{\tau_{i j}^{\prime} \tilde{S}_{i j}^{\prime}}=\overline{\tau_{i j} \tilde{S}_{i j}}-\overline{\tau_{i j}} \overline{\tilde{S}_{i j}}
$$

The two terms in the right-hand side are referred to as $-\epsilon_{\Delta}$ and $-\epsilon_{m}$, respectively. $-\epsilon_{\Delta}$ is usually called the SGS dissipation rate of the kinetic energy. $-\epsilon_{m}$ represents the energy dissipation directly from the mean flow.

The shear production $P_{s}$, the dissipation rate $-\epsilon_{f}$, and the work against the canopy drag $W_{d}$ are plotted in Fig. 14 as a function of height. $W_{d}$ is not available in the PIV data. The dissipation rate $-\epsilon$ from the PIV data is estimated by fitting a $-5 / 3$ slope line to the distribution of one-dimensional longitudinal energy spectra in the inertial range [49]. This dissipation rate $-\epsilon$ determined from the PIV energy spectrum can also be regarded as the 'spectral energy flux' across the scales since it is determined from cascade arguments associated with the spectrum.

There is very good agreement between the LES predictions and the PIV data for the shear production. The $P_{S}$ profile shows a large peak at the top of the stalks where the strong wind shear occurs. $P_{S}$ decreases sharply within the stalks, and is nearly zero below $z / h=0.5$. $P_{s}$ shows another peak near the upper wall due to the large shear at the upper wall boundary layer, and approaches zero at $z / h=2.7$, corresponding to the location of zero mean shear in Fig. 3, and also the lowest turbulence intensity above the stalks shown in Figs. 8 and 9. This 


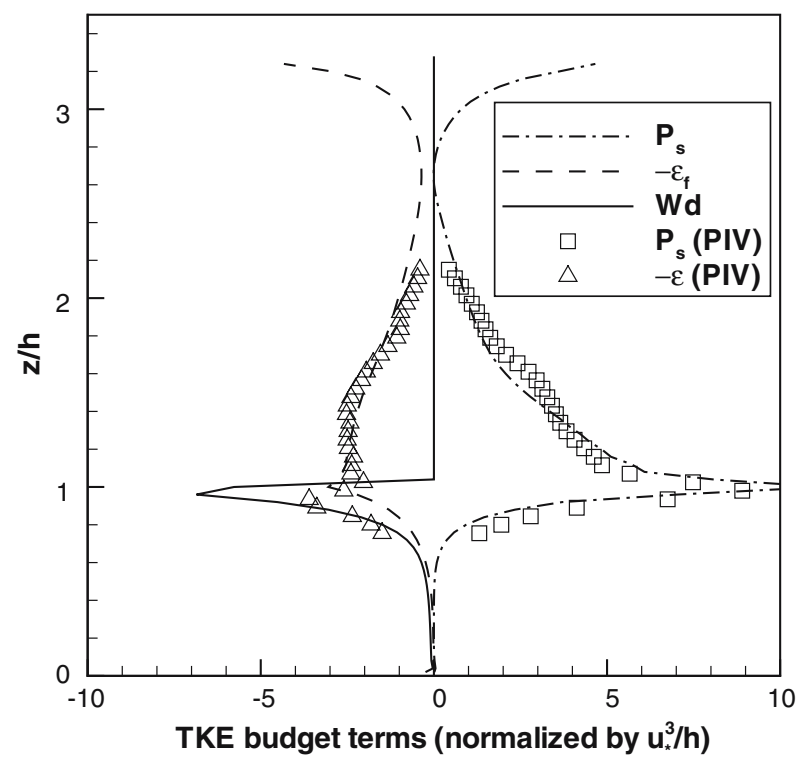

Fig. 14 Turbulent kinetic energy budget terms: shear production and dissipation

is also the location where the Reynolds shear stress (also correlation coefficient) vanishes, as shown in Figs. 10 and 11.

As far as the dissipation profiles are concerned, $-\epsilon_{f}$ shows peak magnitudes at the top of the stalks and near the upper wall, and has the same sharp decreasing trend inside the stalks, as seen with $P_{s}$. Below $z / h=1.6,-\epsilon_{f}$ is considerably smaller than $P_{s}$ in magnitude. Above the stalks, the agreement between the dissipation rate of the PIV and LES data is quite good. This is consistent with the view that the SGS dissipation rate (the LES calculation) is close to the energy flux (the PIV estimation) when the filter size falls into the inertial range [22]. However, inside the canopy, the SGS dissipation rate is smaller than the energy flux evaluated from the PIV spectra. This difference may be caused by the spectral bypass process (see [10] for a detailed discussion), i.e., eddies larger than canopy elements may lose TKE to the wake kinetic energy in addition to the energy cascading process. Examining the differences in more detail, we recall that Zhu et al. [49] have measured the SGS dissipation directly from spatially filtering the PIV data and evaluating the contraction of measured SGS stress and filtered strain-rate tensors (see their Table 1). For instance, at $z / h=0.81$, they observe that the SGS dissipation is $38 \%$ lower than the dissipation obtained from the fit through the measured energy spectrum. Consistent with this direct experimental result, as seen in Fig. 14, below $z / h=1$ the LES yields an SGS dissipation which is lower than the dissipation estimated from the fit through the measured energy spectra. We further remark that this trend also is consistent with the argument presented in [10], that for wind-tunnel canopies the dissipation estimated by spectral fit may overestimate the true rate of energy flux in the cascade process by about one-third.

The rate of work term, $W_{d}$, which in the LES exists within the canopy, attains its maximum near the top of the stalks, as a direct result of the large drag and velocity occurring at the stalk top, and diminishes rapidly inside the model canopy. $W_{d}$ exceeds the dissipation rate, indicating that the canopy drag is the major mechanism suppressing turbulent motions (of scales larger than our numerical grid size) in the canopy. The same conclusion is also 


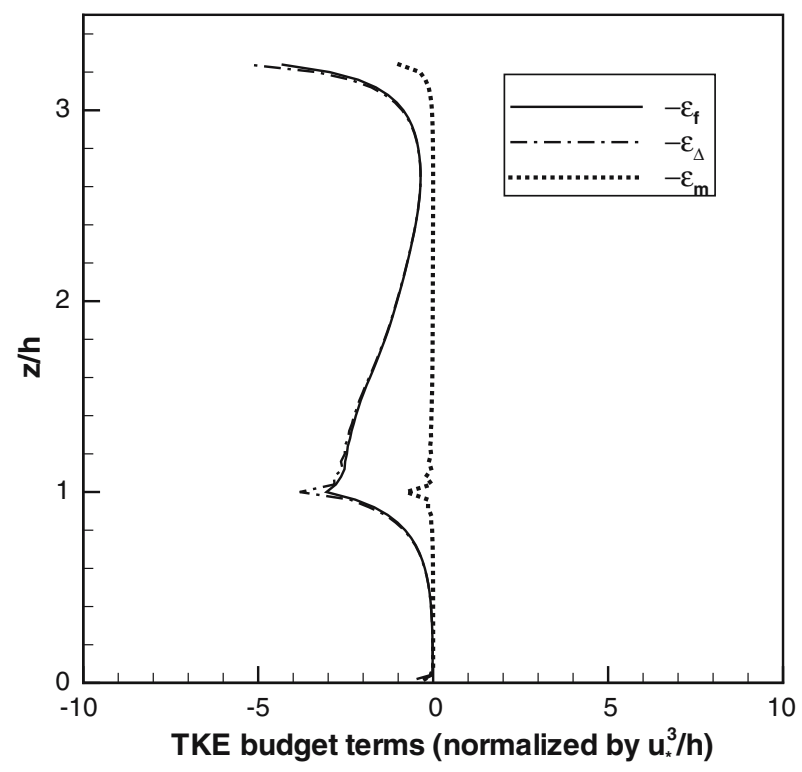

Fig. 15 Turbulent kinetic energy: dissipation decomposition

obtained in [43] based on RANS simulations and in [36], based on LES. The decomposed dissipation rate components are shown in Fig. 15. The energy dissipation directly from the mean flow $-\epsilon_{m}$ is negligible except at the canopy top and the upper wall. The SGS dissipation rate, $-\epsilon_{\Delta}$, can be thus considered as a good estimate for the TKE dissipation rate $-\epsilon_{f}$ over almost the entire flow.

The three transport terms, $T_{t}, T_{p}$, and $T_{\text {sgs }}$, are plotted in Fig. 16. Since the spanwise velocity fluctuations are not measured in the PIV, the turbulent kinetic energy $K$ is estimated as $0.75\left(u^{\prime 2}+w^{\prime 2}\right)$ using the PIV data. Both the LES and the PIV profiles show that $T_{t}$ changes signs around the top of the stalks, indicating that turbulence extracts energy from the free shear flow immediately above the model canopy $(1<z / h<1.5)$ and redistributes the energy into the model canopy $(z / h<1)$ and the upper air flow region $(z / h>1.5)$. The pressure transport is of interest because a direct experimental measurement of pressure is very difficult. $T_{p}$ demonstrates a similar behavior to $T_{t}$ inside and immediately above the model canopy but with smaller magnitudes. Above $z / h=1.2, T_{p}$ is opposite to $T_{t}$ in sign. Pressure fluctuations extract energy from the air layer $1<z / h<1.2$ and transport it into the model canopy and the upper air layer $1.2<z / h<1.6$. Above $z / h=1.6, T_{p}$ is negative again but small in magnitude. The SGS transport $T_{\mathrm{sgs}}$ is insignificant compared to $T_{t}$ and $T_{p}$, except around the top of the stalks where the largest stress fluctuations occur. In general, $T_{\mathrm{sgs}}$ follows the same trend as $T_{t}$ and $T_{p}$ : negative below and positive above the top of the model canopy. Approaching the upper wall, the transport terms are much smaller than $P_{s}$ and $\epsilon_{f}$, and consequently $P_{s}$ and $-\epsilon_{f}$ basically counterbalance each other, achieving the so-called the state of local equilibrium.

As shown in Fig. 17, deep inside the model canopy $(z / h<0.4)$, all three transport terms are small in magnitude compared to the values near the canopy and the free shear layer above it. $P_{s}$ also nearly vanishes due to the low mean shear deep inside the model canopy (see Fig. 3). However, in relative terms $T_{p}$, acts as the largest TKE supplier in this layer. A similar 


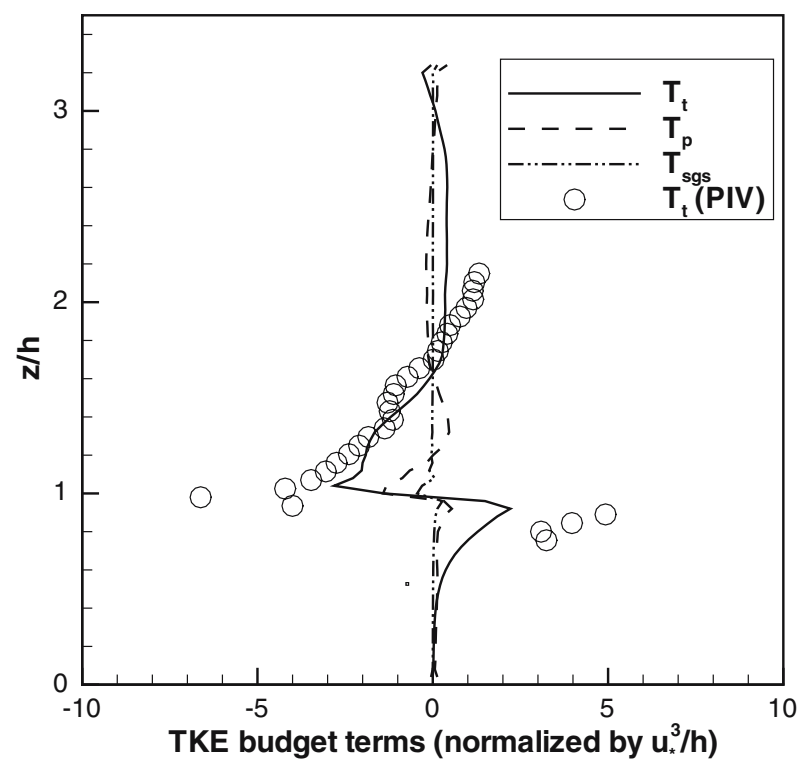

Fig. 16 Turbulent kinetic energy budget terms: turbulent transport $\left(T_{t}\right)$, pressure transport $\left(T_{p}\right)$, and SGS transport $\left(T_{\mathrm{Sgs}}\right)$

result has been previously reported in [35] in terms of lagged cross-correlation analysis of experimental data in a forest, and in [8] in terms of LES data. They also concluded that turbulence at the lower levels of the canopy, dominated by inactive "sloshing" motions, as discussed before, is largely driven by pressure fluctuations [15]. The work against the stalk drag $W_{d}$ is the largest TKE sink away from the bottom wall. Very close to the bottom wall, $\epsilon_{f}$ becomes the largest TKE sink, and $T_{\text {sgs }}$ is second to it. $T_{p}$ also increases sharply near the wall as a counterbalance.

In Fig. 18, the residual $R_{S}$ (the sum of the right hand side of Eq. 12) is plotted. $P_{S}$ is also shown in this figure for comparison. The residual shows some non-zero peaks around the top of the stalks and the upper wall, where strong shear and gradients of flow quantities occur. These peaks are mainly the result of numerical errors. In the original filtered Navier-Stokes equations Eq. 1, the flow variables are defined on the staggered grid, e.g., $\tilde{u}, \tilde{v}, \tilde{p}$, and $\tau_{x y}$ are defined at positions of $(j-1 / 2) \Delta z$ (called $u v$-nodes), where $j=1,2, \ldots, N_{z}$. Some other flow variables, e.g., $\tilde{w}, \tau_{x z}$ and $\tilde{S}_{m n}$, where $m, n=x, y$, and $z$, are defined at positions of $j \Delta z$ (called $w$-nodes). All the variables on the $u v$-nodes in the TKE Eq. 12 are interpolated to the $w$-nodes using linear interpolation. This interpolation causes no significant error at locations where the flow variables change smoothly, but this is not the case at locations where the flow variables change sharply, e.g., at the canopy top and near the upper wall.

\section{Conclusions}

Turbulent flow within and over a wind-tunnel model canopy composed of stalks has been investigated using LES. Different from previous large eddy simulations of canopy flows, we have used a plant-scale representation of the stalks and a scale-dependent Lagrangian dynamic SGS model. The turbulence statistics and turbulent kinetic energy budgets predicted by the 


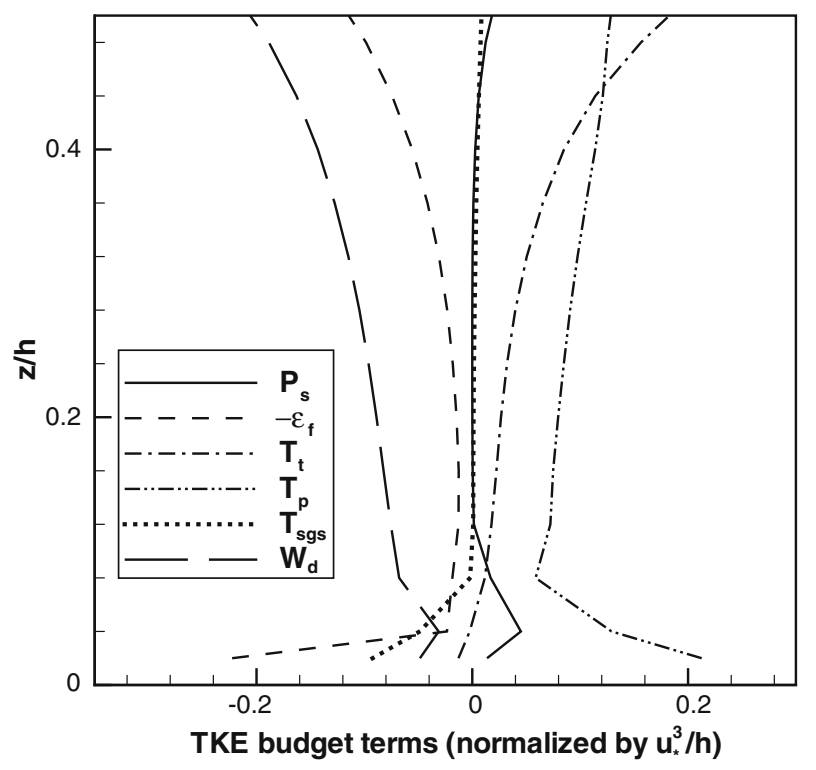

Fig. 17 Turbulent kinetic energy budget terms in the lower half of the model canopy

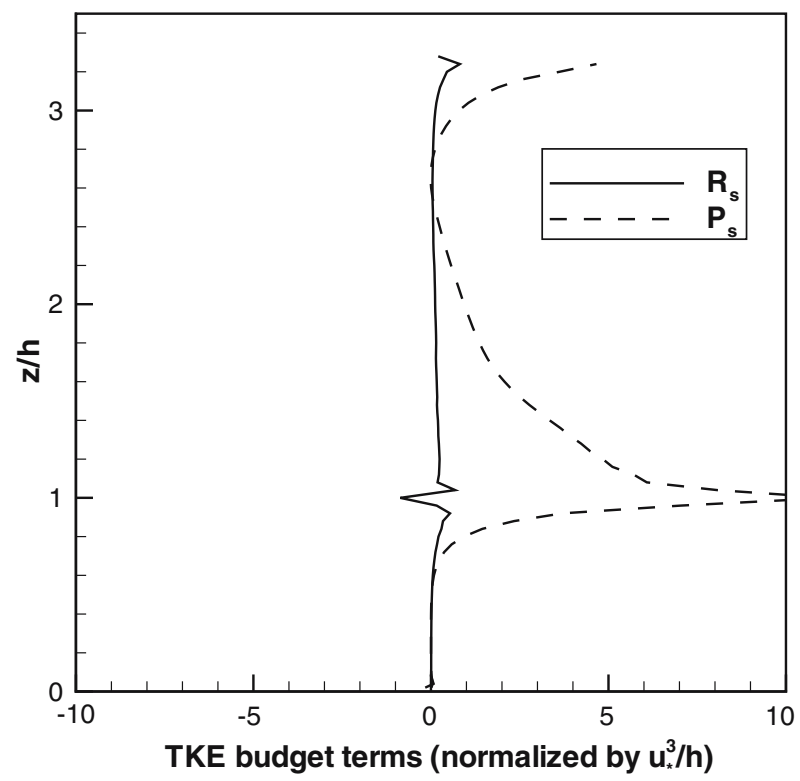

Fig. 18 Turbulent kinetic energy budget terms: residual $\left(R_{S}\right)$ and shear production $\left(P_{S}\right)$

LES have been compared with those of the PIV and hot-wire anemometry experimental data obtained in a wind-tunnel. For most quantities good agreement between the LES results and the experimental data has been obtained. Some differences in the mean velocity profile away from the canopy top exist due to differences in inflow boundary conditions, but turbulence quantities such as root-mean-square profiles, flatness factors, and spectra as function 
of streamwise wavenumber show generally good agreement. These results provide a further validation of the numerical model, in addition to those already presented in [46].

In terms of physics of canopy turbulence, the LES results have led to a confirmation of many findings reached in the prior literature that have been obtained under rather different conditions: field experiments in corn fields [37,41,44], forests [13,38], and LES using homogeneously distributed force fields $[8,27,39,40]$ ("field-scale" representation). Among these findings are the relatively high turbulence intensity inside the canopy, the presence of Kelvin-Helmholtz-like coherent structures near the top of the canopy, as well as the presence of inactive sloshing motions near the ground. The LES has also displayed a difference between the energy flux obtained from the energy spectrum and the SGS dissipation rate. This appears to confirm the existence of a spectral bypass mechanism [10] in which the real spectral flux due to cascade is less than that implied by the energy-spectrum level since part of the energy is absorbed by the interactions with the canopy (modeled in LES as a body force and thus leading to a rate of work). The detailed examination of the TKE budgets has confirmed two important conclusions in canopy turbulence. First, the sloshing motions at the low levels of the canopy are mainly driven by the pressure fluctuations $[15,35]$. Second, the rate of work against the canopy drag is large and can exceed the dissipation rate of kinetic energy $[36,43]$. Taken together, the robustness of these observations is encouraging since the existence of universal features of canopy turbulence should lead to more accurate models and improved understanding.

Acknowledgements This study was supported by the National Science Foundation under Grant No. BES0119903. Discussions with Roger Shaw are gratefully acknowledged.

\section{References}

1. Albertson JD, Parlange MB (1999) Natural integration of scalar fluxes from complex terrain. Adv Water Resour 23:239-252

2. Albertson JD, Parlange MB (1999) Surface length-scales and shear stress: implications for landatmosphere interaction over complex terrain. Water Resour Res 35:2121-2132

3. Amiro BD (1990) Drag coefficients and turbulence spectra within three boreal forest canopies. BoundLayer Meteorol 52:227-246

4. Bou-Zeid E, Meneveau C, Parlange MB (2005) A scale-dependent Lagrangian dynamic model for large eddy simulation of complex turbulent flows. Phys Fluids 17:025105

5. Bradley EF, Finnigan JJ (1973) Heat and mass transfer in the plant-air continuum. In: Proc. First Australasian Heat and Mass Conference. Monash Univ, Melbourne, Australia, pp 57-76

6. Bradshaw P (1967) 'Inactive' motion and pressure fluctuations in turbulent boundary layers. J Fluid Mech 30:241-258

7. Brunet Y, Finnigan JJ, Raupach MR (1994) A wind-tunnel study of air flow in waving wheat: singlepoint velocity statistics. Bound-Layer Meteorol 70:95-132

8. Dwyer MJ, Patton EG, Shaw RH (1997) Turbulent kinetic energy budgets from a large-eddy simulation of airflow above and within a forest canopy. Bound-Layer Meteorol 84:23-43

9. Finnigan JJ (1979) Turbulence in waving wheat I. Mean statistics and honami. Bound-Layer Meteorol $16: 181-211$

10. Finnigan JJ (2000) Turbulence in plant canopies. Annu Rev Fluid Mech 32:519-571

11. Fitzmaurice L, Shaw RH, Paw UKT, Patton EG (2004) Three-dimensional scalar microfront system in a large-eddy simulation of vegetation canopy flow. Bound-Layer Meteorol 112:107-127

12. Gao W, Shaw RH, Paw UKT (1989) Observation of organized structure in turbulent flow within and above a forest canopy. Bound-Layer Meteorol 47:349-377

13. Gardiner BA (1994) Wind and wind forces in a plantation spruce forest. Bound-Layer Meteorol 67:161186

14. Germano M, Piomelli U, Moin P, Cabot W (1991) A dynamic subgrid-scale eddy viscosity model. Phys Fluids A 3:1760-1765 
15. Holland JZ (1989) On pressure-driven wind in deep forests. J Appl Meteorol 28:1349-1355

16. Kaimal JC, Finnigan JJ (1994) Atmospheric boundary layer flows: their structure and mesurement. Oxford University Press, New York

17. Kanda M, Hino M (1994) Organized structures in developing turbulent flow within and above a plant canopy using a large eddy simulation. Bound-Lay Meteorol 68:237-257

18. Kang HS, Chester S, Meneveau C (2003) Decaying turbulence in an active-grid-generated flow and comparison with large-eddy simulation. J Fluid Mech 480:129-160

19. Lilly DK (1992) A proposed modification of the Germano subgrid-scale closure method. Phys Fluids A 4:633-635

20. Jacob M (1938) Heat transfer and flow resistence in cross flow of gases over tube banks. Trans ASME 59:384-386

21. Meneveau C, Katz J (2000) Scale-invariance and turbulence models for large-eddy simulation. Annu Rev Fluid Mech 32:1-32

22. Meneveau C, Lund T, Cabot W (1996) A Lagrangian dynamic subgrid-scale model of turbulence. J Fluid Mech 319:353-385

23. Meroney RN (1968) Characteristics of wind and turbulence in and above model forests. J Appl Meteorol 7:780-788

24. Moeng CH (1984) A large-eddy simulation model for the study of planetary boundary-layer turbulence. J Atmos Sci 41:2052-2062

25. Moeng CH, Wyngaard JC (1989) Evaluation of turbulent transport and dissipation closures in secondorder modeling. J Atmos Sci 46:2311-2330

26. Orszag SS (1970) Transform method for calculation of vector coupled sums: application to the spectral form of vorticity equation. J Atmos Sci 27:890-895

27. Patton EG, Shaw RH, Judd MJ, Raupach MR (1998) Large-eddy simulation of windbreak flow. BoundLayer Meteorol 87:275-306

28. Poggi D, Porporato A, Ridolfi L, Albertson JD, Katul GG (2004) The effect of vegetation density on canopy sub-layer turbulence. Bound-Layer Meteorol 111:565-587

29. Porté-Agel F, Meneveau C, Parlange MB (2000) A scale-dependent dynamic model for large-eddy simulation: application to a neutral atmospheric boundary layer. J Fluid Mech 415:261-284

30. Raupach MR, Thom AS (1981) Turbulence in and above plant canopies. Annu Rev Fluid Mech 13:97129

31. Raupach MR, Coppin PA, Legg BJ (1986) Experiments on scalar dispersion within a model plant canopy. Part I: The turbulence structure. Bound-Layer Meteorol 35:21-52

32. Roshko A (1961) Experiments on the flow past a circular cylinder at very high reynolds number. J Fluid Mech 10:345-356

33. Seginer I, Mulhearn PJ, Bradley EF, Finnigan JJ (1976) Turbulent flow in a model plant canopy. BoundLayer Meteorol 10:423-453

34. Shaw RH, Schumann U (1992) Large-eddy simulation of turbulent flow above and within a forest. Bound-Layer Meteorol 61:47-64

35. Shaw RH, Zhang XJ (1992) Evidence of pressure-forced flow in a forest. Bound-Layer Meteorol 58:273288

36. Shaw RH, Patton EG (2003) Canopy element influences on resolved- and subgrid scale energy within a large-eddy simulation. Agric For Meteorol 115:5-17

37. Shaw RH, Silversides RH, Thurtell GW (1974) Some observations of turbulence and turbulent transport within and above plant canopies. Bound-Layer Meteorol 5:429-449

38. Shaw RH, Den Hartog G, Neumann HH (1988) Influence of foliar density and thermal stability on profiles of Reynolds stress and turbulence intensity in a deciduous forest. Bound-Layer Meteorol 45:391-409

39. Shen S, Leclerc MY (1997) Modeling the turbulence structure in the canopy layer. Agric For Meteorol $87: 3-25$

40. Su HB, Shaw RH, Paw UKT, Moeng CH, Sullivan PP (1998) Turbulent statistics of neutrally stratified flow within and above a sparse forest from large-eddy simulation and field observations. Bound-Layer Meteorol 88:363-397

41. van Hout R, Zhu W, Luznik L, Katz J, Kleissl J, Parlange MB (2007) PIV measurements in the atmospheric boundary layer within and above a mature corn canopy. Part A: Statistics and small scale isotropy. J Atmos Sci 64:2805-2824

42. Wilson JD (1988) A second-order model for flow through vegetation. Bound-Layer Meteorol 42:371392

43. Wilson NR, Shaw RH (1977) A higher order closure model for canopy flow. J Appl Meteorol 16:11971205 
44. Wilson JD, Ward DP, Thurtell GW, Kidd GE (1982) Statistics of atmospheric turbulence within and above a corn canopy. Bound-Layer Meteorol 24:495-519

45. Yue W, Meneveau C, Parlange MB, Zhu W, van Hout R, Katz J (2007) A comparative quadrant analysis of turbulence in a plant canopy. Water Resour Res 43: W05422, DOI:10.1029/2006WR005583

46. Yue W, Parlange MB, Meneveau C, Zhu W, van Hout R, Katz J (2007) Large eddy simulation study of turbulence structures within and above a corn canopy, using field- and plant-scale representations. Bound-Layer Meteorol 124:183-203

47. Zhu W, van Hout R, Luznik L, Kang HS, Katz J, Meneveau C (2006) A comparison of PIV mesurements of canopy turbulence performed in the field and in a wind-tunnel model. Exp Fluids 41:309-318

48. Zhu W, van Hout R, Katz J (2007) PIV measurements in the atmospheric boundary layer within and above a mature corn canopy. Part B: Quadrant-hole analysis. J Atmos Sci 64:2825-2838

49. Zhu W, van Hout R, Katz J (2007) On the flow structure and turbulence during sweep and ejection events in a model canopy. Bound-Layer Meteorol 124:205-233 